\title{
Floristic and structural comparisons between woody communities of two seasonal forest fragments in the Tocantins river basin and other remnants of this forest physiognomy in Brazil
}

Comparações florísticas e estruturais entre comunidades arbóreas de dois fragmentos de florestas estacionais na bacia do rio Tocantins e outros remanescentes desta fisionomia florestal no Brasil

\author{
Marcelo Brilhante de Medeiros ${ }^{1,3}$, Bruno Machado Teles Walter $^{1}$ \& Washington Luis Oliveira ${ }^{2}$
}

\begin{abstract}
This work describes the woody layer composition and structure in two seasonal forest fragments in the Tocantins river basin and compares them to other remnants of this forest physiognomy in Brazil. The survey was carried out by using 17 plot samples $(20 \times 50 \mathrm{~m})$ located in Palmeirópolis, state of Tocantins, and in Minaçú, state of Goiás. All woody individuals showing diameters $\geq 5 \mathrm{~cm}$, at $1.30 \mathrm{~cm}$ above ground level, were recorded. The higher floristic similarity of these forest remnants compared with other closer seasonal forests did not show a distinct pattern. The floristic composition was more similar to that of a deciduous seasonal forest in the Paranã valley, and more dissimilar to other forests in this same valley. This result and a higher dissimilarity related to the southern forests in Goiás suggest that the forest fragments showed a floristic composition and structure typical of lowland seasonal forests in the Tocantins river basin. The results also indicated that the forest fragments have distinct floristic compositions with a relatively similar structure and diversity.
\end{abstract}

Key words: dry seasonal forests, diversity, composition.

\section{Resumo}

A composição e a estrutura do componente arbóreo foram caracterizadas em dois fragmentos de floresta estacional na bacia do médio rio Tocantins e comparadas com outros remanescentes dessa fitofisionomia em outras regiões do Brasil. Os indivíduos foram amostrados em 17 parcelas de $20 \times 50 \mathrm{~m}$, nos municípios de Palmeirópolis (TO) e Minaçú (GO). Em cada parcela foram mensurados todos os indivíduos lenhosos que apresentaram diâmetro igual ou superior a $5 \mathrm{~cm}$, medido a $1,30 \mathrm{~m}$ do solo (DAP $\geq 5 \mathrm{~cm}$ ). A maior similaridade florística desses dois remanescentes com outras florestas estacionais mais próximas não apresentou um padrão claro. A composição florística nos fragmentos estudados foi mais similar a uma floresta estacional decidual no vale do Paranã, embora muito dissimilar em relação à outra floresta decidual nesse mesmo vale. Esse resultado e a maior dissimilaridade em relação às florestas do sul de Goiás sugerem que as atuais florestas apresentaram composição e estrutura características de florestas estacionais de baixa altitude da bacia do médio/alto rio Tocantins. Os resultados também indicam que cada fragmento investigado apresenta uma flora própria, com estrutura e diversidade relativamente similares.

Palavras-chave: mata seca, diversidade, composição.

\section{Introduction}

From 2000 to 2010 tropical deforestation due to agricultural development showed signs of falling in a number of tropical countries, although the scale of this activity continues to cause concern (CDB 2010). During this time about $130,000 \mathrm{~km}^{2}$ of forests were converted into other land uses each year and the data from the past decade showed deforestation rates of 160,000 km².year-1 (CDB 2010).

Seasonal forests, also known as dry forests, deciduous or semi-deciduous forests, are often considered the most endangered types of tropical

\footnotetext{
'Embrapa Recursos Genéticos e Biotecnologia, Parque Estação Biológica, Av. W5 Norte (Final) s/nº, Brasília, DF, 70770-917, Brazil.

${ }^{2}$ Universidade de Brasília, Depto. Ecologia, Curso de Pós-graduação em Ecologia, Campus Universitário Darcy Ribeiro, Brasília, DF, 70910-900, Brazil.

${ }^{3}$ Author for correspondence: marcelo.brilhante@embrapa.br
} 
forests because of the widespread fragmentation and area reduction caused by agriculture, logging of selected woods and mining (Janzen 1997; Scariot \& Sevilha 2005; Espírito-Santo et al. 2009). It is common for seasonal forest to undergo disturbances such as fire and invasive species (Janzen 1997). In addition to these impacts, conservation efforts in recent decades have been directed toward tropical rain forests, and less attention has been paid to dry forests (Vieira \& Scariot 2006a). This type of vegetation had estimated annual deforestation rates between 1981 and 1990 of $0.94 \%$.year $^{-1}$, or 61 million hectares. year $^{-1}$. On the other hand, tropical rain forests showed lower deforestation rates of $0.64 \%$.year ${ }^{-1}$ or 46 million hectares.year ${ }^{-1}$ (Whitmore 1997).

Knowledge about the floristic distribution patterns of seasonal forests could provide valuable information for conservation actions (Scariot \& Sevilha 2000). The highest values of similarity of seasonal forests have been associated with the influence of nearby vegetation formations in northeastern and central Brazil (Garcia et al. 2011; Santos et al. 2012). However, there is a lack of studies comprising the seasonal forests in some regions of central Brazil such as the "mato grosso goiano" in northeastern Goiás and southern Tocantins.

The valley of the middle Tocantins river, located between the municipalities of Palmeirópolis, in the state of Tocantins (TO), and Minaçú, in Goiás (GO), has few and small seasonal forest remnants in an extensive agricultural landscape planted with exotic grasses for grazing. This region in central Brazil comprises the northeastern edge of the former forest swathe called the "mato grosso goiano", which originally covered large areas of the state of Goiás, from the south, through the valley of the Paranaíba river and stretching north to the valley of the Paranã river and some areas of the middle Tocantins (Faissol 1953; Veloso 1963). This forest was converted into an intensive agriculture landscape throughout the $20^{\text {th }}$ century in a south-north direction, and nowadays these forest remnants are just disconnected fragments.

The aim of this study was to characterize the floristic composition and horizontal structure of the woody layer of two forest fragments in the region of the former "mato grosso goiano", in the middle Tocantins river basin, and to compare them to other remnants of this forest physiognomy in Brazil. The study also aims to evaluate the influence of nearby seasonal forests on the floristic distribution and structural patterns of these fragments in this region in order to provide information about the community ecology of these highly endangered seasonal forests.

\section{Material and Methods}

Study area

The two semi-deciduous seasonal forest fragments were sampled in the municipalities of Palmeirópolis-TO (130' $47^{\circ}$ "S and 48 $\left.8^{\circ} 13^{\prime} 13^{\prime \prime} \mathrm{W}\right)$ and Minaçú-GO $\left(13^{\circ} 18^{\prime} 44^{\prime \prime} \mathrm{S}\right.$ and $\left.48^{\circ} 10^{\prime} 45^{\prime \prime} \mathrm{W}\right)$ (Figure 1). These forest fragments are inside a large belt of intensive agriculture with exotic grasses and some native remnants with savanna vegetation.

The climate in this region is classified as Aw in accordance with Köppen, which means wet tropical with two well marked climatic seasons (dry winters and wet summers). The mean annual rainfall is from 1,400 to $1,800 \mathrm{~mm}$ and the mean annual temperature is from 23 to $25^{\circ} \mathrm{C}$ (Silva et al. 2008). The altitude of the fragments is between 300 and $370 \mathrm{~m}$ and the relief is from flat to smooth slopes located in the Tocantins valley, Tocantins middle river basin (BRASIL 1981). In Palmeirópolis, the plots were located on a flat site and in Minaçú the plots lay on a smooth slope.

The forest fragments are classified as submountainous semi-deciduous seasonal forest in accordance with IBGE vegetation classification (IBGE 2012). In accordance with Ribeiro \&

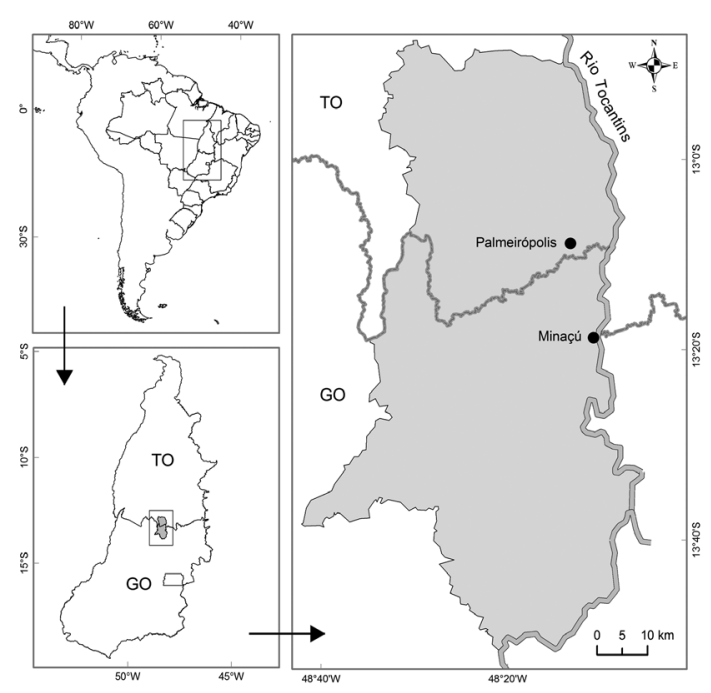

Figure 1 -Study area in the municipalities of the states of Goiás (GO) and Tocantins (TO), Brazil. 
Walter (2008) these forests would be classified as semi-deciduous dry forests. The forests are not associated with bodies of water, and in the Cerrado biome three subtypes can be found (evergreen, semi-deciduous and deciduous), which are different because of the amount of foliage that persists in the dry season, the floristic composition and the soil type (Ribeiro \& Walter 2008).

The most common soil type in both fragments is argisols, in addition to some sites with lithosols and red latosols (SEPLAN 2008; SIEG 2010). Soils such as the argisols of the Tocantins river basin are related to high levels of $\mathrm{Ca}$ and $\mathrm{Mg}$ in areas of seasonal forests (Scariot \& Sevilha 2005).

The study areas were fragmented, with common evidence of human disturbances typical of this region. These human disturbances comprise agriculture, grazing, fire and logging, and the sites showed an abundance of lianas, bamboo invasion (Guadua paniculata Munro frequent) and exotic trees such as Citrus.

\section{Vegetation survey}

The vegetation was surveyed during the wet season in November 2009. Seventeen permanent plots $(20 \times 50 \mathrm{~m})$ were randomly allocated, 10 plots were set up in Palmeirópolis and seven plots in Minaçú because of the smaller area in the latter site.

In each plot all the woody and palm individuals were sampled if their diameter was at least $5 \mathrm{~cm}$ at $1.30 \mathrm{~cm}$ from soil height $(\mathrm{dbh} \geq 5 \mathrm{~cm})$, measured using a caliper, and their height was estimated by visual perception. Woody lianas were not included in this sampling and were considered only for analysis as a disturbance indicator, as explained below. The species identification was performed in the field surveys and in herbarium. The floristic survey follows the Angiosperm Phylogeny Group III (2009). Vouchers were incorporated into the Embrapa Recursos Genéticos e Biotecnologia herbarium (CEN herbarium).

A dissimilarity qualitative analysis was carried out by using the UPGMA (unweighted pair grouping method using arithmetic averages) linkage method and by Sørensen index (Legendre \& Legendre 2012) regarding the selected fragments and literature data of seasonal forests in Goiás (Silva \& Scariot 2003; Haidar et al. 2005; Nascimento et al. 2004; Imaña-Encinas et al. 2007; Garcia et al. 2011). The phytosociological parameters of horizontal structure (density, frequency, dominance and value of importance) were calculated for the woody communities in each fragment by using the Mata Nativa 2 software (CIENTEC 2004). The floristic diversity was calculated by using the Shannon index (H') with $\log n$ and the evenness was calculated by the Pielou (J') index (Magurran \& McGill 2011). Rarefaction (sample based) was used to compare the richness between the fragments in accordance with Gotelli \& Colwell (2001). Three richness estimators (Chao 1, Jackknife 1 and 2 and Bootstrap) were used to evaluate the number of non-sampled species in addition to the sampled richness in each fragment (Palmer 1990). The analyses were performed by using the statistical environment R ( R Development Core Team 2011), with functions available from Vegan package (Oksanen et al. 2011).

In order to characterize the liana tangles, a variable used as human disturbance indicator (Oliveira-Filho et al. 1997), eight sub-samples of $25 \mathrm{~m}^{2}$ were taken from an area of $20 \times 10 \mathrm{~m}$ allocated randomly to each plot. Each liana tangle represented a set of at least four lianas in each subsample, even if the roots were inside or outside the sub-sample. A diameter at breast height $(\mathrm{DBH}) \geq 3 \mathrm{~cm}$ was established to record the trunks, excluding ferns, Araceae and small vines (Oliveira-Filho et al. 1997).

\section{Results}

Similarity

The UPGMA dendrogram (Fig. 2) showed that the fragments in Palmeirópolis and Minaçú have a floristic composition that is more similar

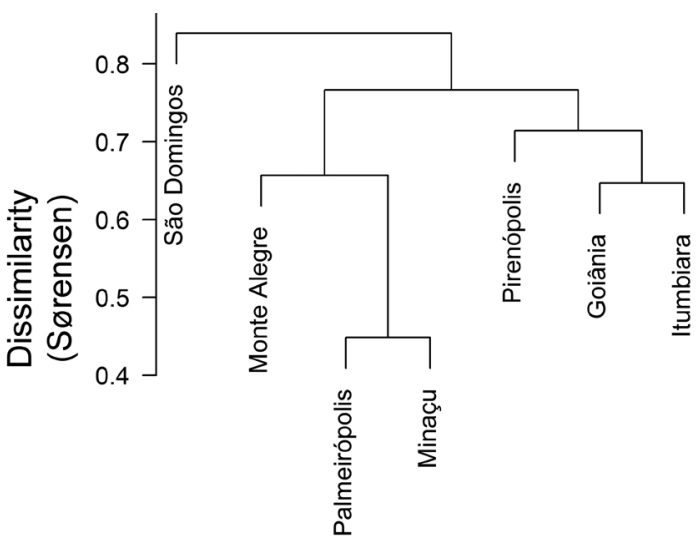

Figure 2 - Dissimilarity dendrogram by using Sorensen index for the woody communities in Palmeirópolis (TO), Minaçu (GO), Goiânia (GO), São Domingos (GO), Monte Alegre (GO), Itaituba (GO) and Pirenópolis (GO). 
to the deciduous seasonal forest of Monte Alegre de Goiás, located in the valley of the Paranã River, in comparison to the other areas. The data from the deciduous forest in the municipality of São Domingos, located in the same valley, showed higher dissimilarity values than those in the current study. In comparison to some seasonal semideciduous forests in central-southern Goiás, the dissimilarity also showed high values, above $70 \%$ in comparison to this study. In fact, Palmeirópolis and Minaçú made a strong cluster in spite of the higher dissimilarity in relation to other studies with seasonal forests in Goiás, including areas of the same semi-deciduous sub-type.

\section{Species richness and diversity}

The data on species richness, Shannon diversity index, Pielou evenness, density, volume, basal area, dead individuals and liana density are described in Table 1. Some parameters commonly used in the phytosociology of other seasonal forests are described in Table 2.

In Palmeirópolis, 31 families, 63 genus and 68 species were sampled (Tab. 3). The ten species with the highest values of importance (Anadenanthera colubrina, Eugenia dysenterica, Aspidosperma subincanum, Dilodendron bipinnatum, Aspidosperma pyrifolium, Myracrodruon urundeuva, Hymenaea courbaril, Bauhinia rufa, Handroanthus impetiginosus and Swartzia acutifolia) comprised $57.8 \%$ of the total importance and $70.9 \%$ of the dominance. In the total of the 837 sampled individuals, 88 (10.5\%) were dead but upright.

In the Minaçú fragment, 23 families, 45 genus and 49 species were sampled (Tab. 4). The ten species with the highest values of importance (Pseudobombax tomentosum, Dilodendron bipinnatum, Tabebuia roseoalba, Anadenanthera colubrina, Luehea paniculata, Magonia pubescens, Callisthene fasciculata, Guettarda viburnoides, Myracrodruon urundeuva and Aspidosperma subincanum) comprised $55 \%$ of the total value of importance and $70.1 \%$ of the value of dominance. Out of the total of 539 sampled individuals in 0.7 ha, $57(10.57 \%)$ were dead individuals, but standing. In considering the density per hectare this percentage persists unaltered $(10.58 \%)$.

\section{Discussion}

The low similarity between areas has been a common pattern in seasonal forests (Pennington et al. 2006), and the results of the dissimilarity analyses indicated distinct composition in relation to the deciduous and semi-deciduous seasonal forests from Goiás. The closer similarity between the semi-deciduous forests of Palmeirópolis and Minaçu in relation to the deciduous seasonal forest of Monte Alegre de Goiás sampled by Nascimento et al. (2004) could suggest the influence of nearby vegetation formations. This was a suggestion made by Garcia et al. (2011) and Santos et al. (2012), without considering the remnants of seasonal forests of the "mato grosso goiano", as performed in this study. However, in the same valley of the Paranã river, the deciduous forest of São Domingos municipality (Silva \& Scariot 2003) showed the most dissimilar fragment in comparison to the current study, which left this analysis inconclusive. Also in a similar position, the semi-deciduous forests from Central and South Goiás (Haidar et al. 2005; Imaña-Encinas et al. 2007; Garcia et al. 2011) were dissimilar to this study. These forests belong to the same sub-type of vegetation as the fragments in this study, increasing the lack of precision regarding any patterns that might emerge. Hence, neither the geographic distances nor the same sub-type of vegetation were enough to explain the patterns of floristic similarity.

The biogeographic patterns of the seasonal forests have suggested a woody flora highly limited by dispersion in remnants of northeastern and central Brazil (Santos et al. 2012). The species observed in the current study, like the floristic studies carried

Table 1 - Patterns of woody communities of seasonal forest fragments in Palmeirópolis (TO) and Minaçú (GO), located in the middle Tocantins river basin. (*) Richness rarefaction values; $(* *)$ Richness estimators: Chao 1; Jackknife ( $1^{\mathrm{a}}$ order); Jackknife ( $2^{\mathrm{a}}$ order); Bootstrap.

\begin{tabular}{lcc}
\hline Parameter & $\begin{array}{c}\text { Palmeirópolis } \\
\text { TO }\end{array}$ & $\begin{array}{c}\text { Minaçú } \\
\text { GO }\end{array}$ \\
\hline Richness of the woody layer & $68(60)^{*}$ & $49(49)^{*}$ \\
Richness estimators** & $111 ; 97 ;$ & $61 ; 63 ;$ \\
Diversity (H') & $113 ; 81$ & $68 ; 55$ \\
Evenness (J') & 3.15 & 3.28 \\
Density (n.ha $\left.{ }^{-1}\right)$ & 0.75 & 0.84 \\
Basal area (m $\left.{ }^{2} . h a^{-1}\right)$ & 749 & 688 \\
Volume (m ${ }^{3} \cdot$ ha $\left.^{-1}\right)$ & 16.5 & 11.9 \\
Dead standing individuals / & 179.37 & 95.6 \\
VI (\%) & $88 / 9.11$ & $57 / 7.23$ \\
Liana tangle frequency & $80 \%$ & $28.5 \%$ \\
\hline
\end{tabular}


Woody communities of two seasonal forests in the Tocantins river basin

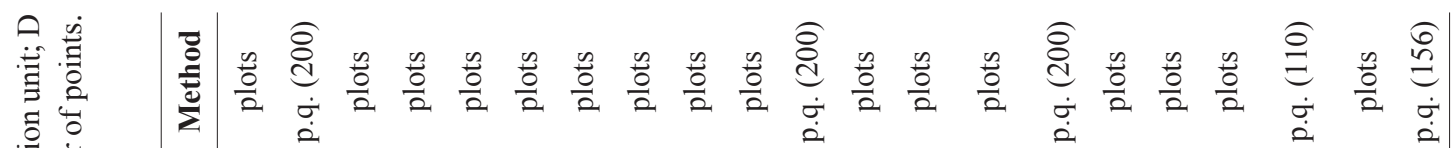

害竞

政

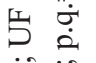

意商

然

咅产

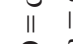

-

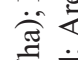

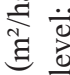

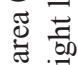

高咅

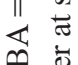

离

䨔

常

을

$\stackrel{\circ}{\ddot{0}}$

$\therefore \frac{2}{2}$

苛

馬

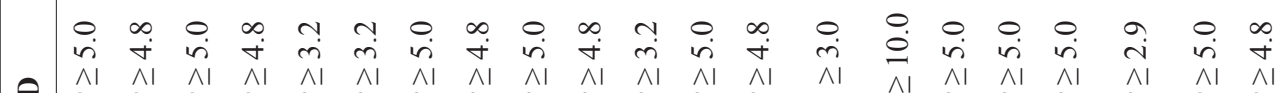

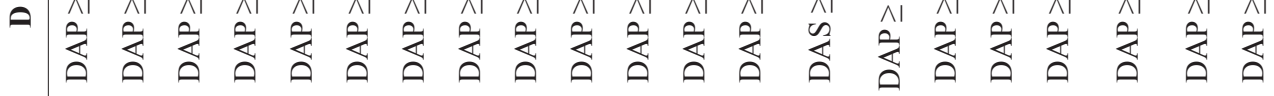
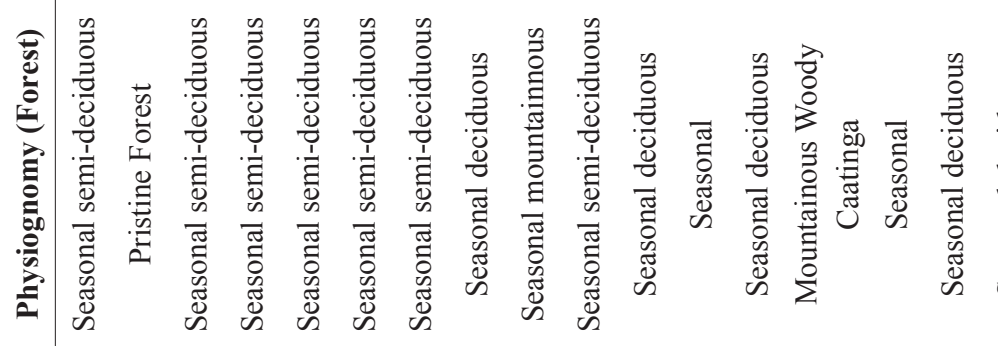

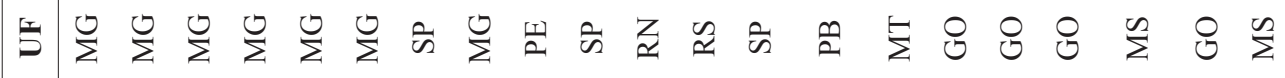

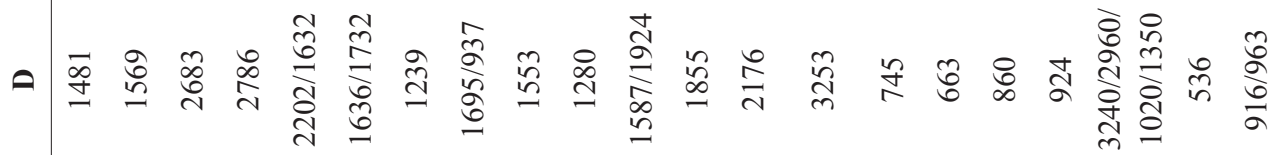

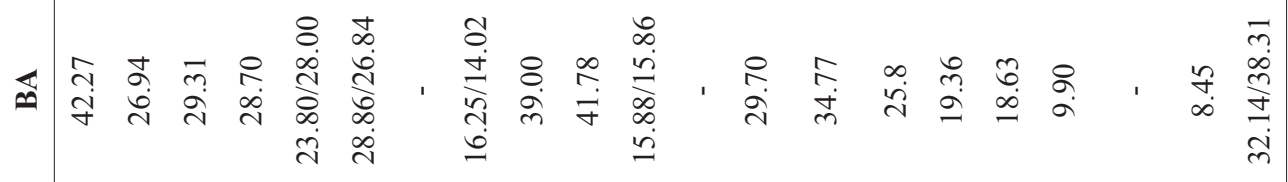

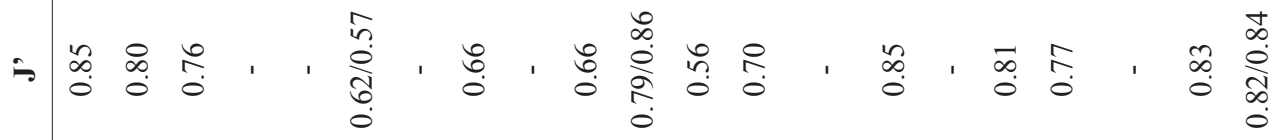

II एँ

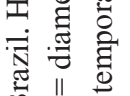

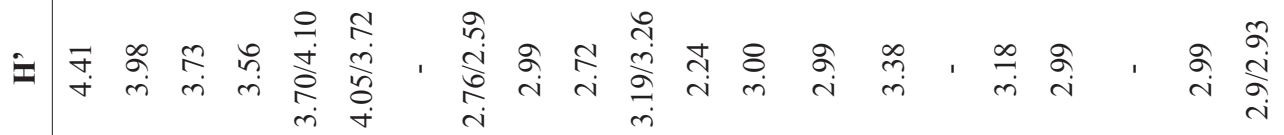

究安主

苟

홍

可 $\Xi$ 逽

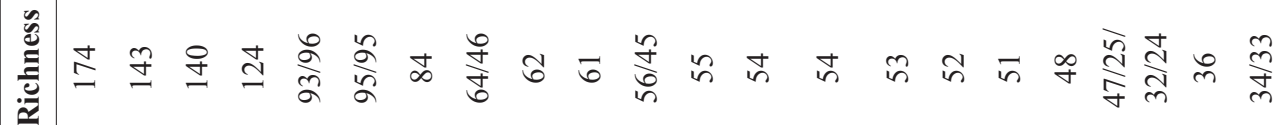

告.

空 言

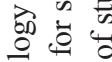

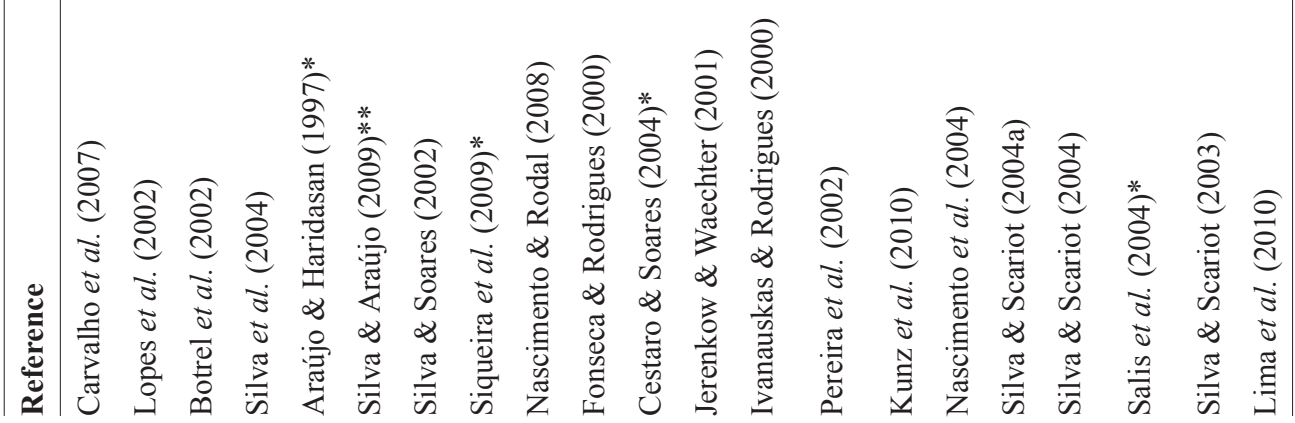


Table 3 - Woody community in one hectare of semi-deciduous seasonal forest in the municipality of Palmeirópolis (TO). Decreasing Values of Importance (VI): D= Density (ind./ha), Do = Dominance ( $\left.\mathrm{m}^{2} / \mathrm{ha}\right), \mathrm{F}=$ Frequency (\%), $\mathrm{A}=$ absolute, $\mathrm{R}=$ relative.

\begin{tabular}{|c|c|c|c|c|c|c|c|c|c|}
\hline Scientific name & Family & DA & DR & FA & FR & DoA & DoR & VI & $\begin{array}{c}\text { VI } \\
(\%)\end{array}$ \\
\hline Anadenanthera colubrina (Vell.) Brenan & Fabaceae & 46.0 & 6.1 & 100.0 & 4.5 & 4.2 & 25.3 & 36.0 & 12.0 \\
\hline Eugenia dysenterica DC. & Myrtaceae & 168.0 & 22.4 & 90.0 & 4.1 & 1.3 & 7.6 & 34.1 & 11.4 \\
\hline Aspidosperma subincanum Mart. & Apocynaceae & 88.0 & 11.8 & 100.0 & 4.5 & 1.1 & 6.6 & 22.9 & 7.6 \\
\hline Dilodendron bipinnatum Radlk. & Sapindaceae & 50.0 & 6.7 & 80.0 & 3.6 & 0.9 & 5.4 & 15.7 & 5.2 \\
\hline Aspidosperma pyrifolium Mart. & Apocynaceae & 39.0 & 5.2 & 90.0 & 4.1 & 0.5 & 3.2 & 12.5 & 4.2 \\
\hline Myracrodruon urundeuva Allemão & Anacardiaceae & 22.0 & 2.9 & 70.0 & 3.2 & 1.0 & 5.9 & 12.0 & 4.0 \\
\hline Hymenaea courbaril L. & Fabaceae & 29.0 & 3.9 & 70.0 & 3.2 & 0.7 & 4.0 & 11.1 & 3.7 \\
\hline Bauhinia rufa (Bong.) Steud. & Fabaceae & 36.0 & 4.8 & 80.0 & 3.6 & 0.3 & 1.8 & 10.2 & 3.4 \\
\hline Handroanthus impetiginosus (Mart. ex DC.) Mattos & Bignoniaceae & 9.0 & 1.2 & 50.0 & 2.3 & 1.1 & 6.7 & 10.2 & 3.4 \\
\hline Swartzia acutifolia Vogel & Fabaceae & 12.0 & 1.6 & 70.0 & 3.2 & 0.7 & 4.3 & 9.1 & 3.0 \\
\hline Agonandra brasiliensis Miers ex Benth. \& Hook.f. & Opiliaceae & 26.0 & 3.5 & 70.0 & 3.2 & 0.3 & 2.1 & 8.7 & 2.9 \\
\hline Tabebuia roseoalba (Ridl.) Sandwith & Bignoniaceae & 18.0 & 2.4 & 80.0 & 3.6 & 0.3 & 1.9 & 7.9 & 2.6 \\
\hline Casearia rupestris Eichler & Salicaceae & 14.0 & 1.9 & 60.0 & 2.7 & 0.2 & 1.0 & 5.6 & 1.9 \\
\hline Terminalia argentea Mart. & Combretaceae & 6.0 & 0.8 & 40.0 & 1.8 & 0.5 & 2.8 & 5.4 & 1.8 \\
\hline Machaerium cf. aculeatum Raddi & Fabaceae & 11.0 & 1.5 & 50.0 & 2.3 & 0.3 & 1.6 & 5.3 & 1.8 \\
\hline Guapira sp.1 & Nyctaginaceae & 9.0 & 1.2 & 70.0 & 3.2 & 0.1 & 0.5 & 4.8 & 1.6 \\
\hline Apeiba tibourbou Aubl. & Malvaceae & 4.0 & 0.5 & 30.0 & 1.4 & 0.5 & 2.9 & 4.8 & 1.6 \\
\hline Callisthene fasciculata Mart. & Vochysiaceae & 5.0 & 0.7 & 50.0 & 2.3 & 0.3 & 1.7 & 4.7 & 1.6 \\
\hline Psidium myrtoides O.Berg & Myrtaceae & 11.0 & 1.5 & 60.0 & 2.7 & 0.1 & 0.3 & 4.5 & 1.5 \\
\hline Rhamnidium elaeocarpum Reissek & Rhamnaceae & 10.0 & 1.3 & 50.0 & 2.3 & 0.1 & 0.8 & 4.3 & 1.5 \\
\hline Sapium glandulosum (L.) Morong & Euphorbiaceae & 9.0 & 1.2 & 40.0 & 1.8 & 0.2 & 1.3 & 4.3 & 1.4 \\
\hline Senegalia polyphylla (DC.) Britton \& Rose & Fabaceae & 11.0 & 1.5 & 50.0 & 2.3 & 0.1 & 0.5 & 4.2 & 1.4 \\
\hline Campomanesia velutina (Cambess.) O.Berg & Myrtaceae & 13.0 & 1.7 & 40.0 & 1.8 & 0.1 & 0.5 & 4.0 & 1.3 \\
\hline Sterculia striata A.St.-Hil. \& Naudin & Malvaceae & 4.0 & 0.5 & 40.0 & 1.8 & 0.2 & 1.2 & 3.5 & 1.2 \\
\hline Magonia pubescens A. St.-Hil. & Sapindaceae & 5.0 & 0.7 & 30.0 & 1.4 & 0.2 & 1.3 & 3.3 & 1.1 \\
\hline Cheiloclinium cognatum (Miers) A.C. Sm. & Celastraceae & 6.0 & 0.8 & 50.0 & 2.3 & 0.0 & 0.2 & 3.2 & 1.1 \\
\hline Guazuma ulmifolia Lam. & Malvaceae & 9.0 & 1.2 & 30.0 & 1.4 & 0.0 & 0.2 & 2.8 & 0.9 \\
\hline Margaritaria nobilis L.f. & Phyllanthaceae & 4.0 & 0.5 & 40.0 & 1.8 & 0.0 & 0.3 & 2.6 & 0.9 \\
\hline Pseudobombax longiflorum (Mart. \& Zucc.) A. Robyns & Malvaceae & 3.0 & 0.4 & 20.0 & 0.9 & 0.3 & 1.8 & 3.1 & 0.9 \\
\hline Syagrus oleracea (Mart.) Becc. & Arecaceae & 5.0 & 0.6 & 30.0 & 1.4 & 0.1 & 0.6 & 2.6 & 0.9 \\
\hline Dipteryx alata Vogel & Fabaceae & 4.0 & 0.5 & 20.0 & 0.9 & 0.1 & 0.7 & 2.1 & 0.7 \\
\hline Tetragastris altissima (Aubl.) Swart & Anacardiaceae & 5.0 & 0.7 & 20.0 & 0.9 & 0.0 & 0.3 & 1.8 & 0.6 \\
\hline
\end{tabular}




\begin{tabular}{|c|c|c|c|c|c|c|c|c|c|}
\hline Scientific name & Family & DA & DR & FA & FR & DoA & DoR & VI & $\begin{array}{l}\text { VI } \\
(\%)\end{array}$ \\
\hline Astronium fraxinifolium Schott ex Spreng. & Anacardiaceae & 3.0 & 0.4 & 20.0 & 0.9 & 0.1 & 0.5 & 1.8 & 0.6 \\
\hline Maclura tinctoria (L.) D. Don ex Steud. & Moraceae & 3.0 & 0.4 & 10.0 & 0.5 & 0.2 & 0.9 & 1.8 & 0.6 \\
\hline Vatairea macrocarpa (Benth.) Ducke & Fabaceae & 3.0 & 0.4 & 20.0 & 0.9 & 0.1 & 0.5 & 1.7 & 0.6 \\
\hline Albizia sp. & Fabaceae & 2.0 & 0.3 & 10.0 & 0.5 & 0.1 & 0.9 & 1.6 & 0.5 \\
\hline Varronia sessilifolia (Cham.) Borhidi & Boraginaceae & 4.0 & 0.5 & 20.0 & 0.9 & 0.0 & 0.1 & 1.5 & 0.5 \\
\hline Erythroxylum daphnites Mart. & Erythroxylaceae & 3.0 & 0.4 & 20.0 & 0.9 & 0.0 & 0.1 & 1.4 & 0.5 \\
\hline Myrcia splendens (Sw.) DC. & Myrtaceae & 3.0 & 0.4 & 20.0 & 0.9 & 0.0 & 0.1 & 1.4 & 0.5 \\
\hline Acrocomia aculeata (Jacq.) Lodd. ex Mart. & Arecaceae & 2.0 & 0.3 & 20.0 & 0.9 & 0.0 & 0.2 & 1.3 & 0.5 \\
\hline Buchenavia tomentosa Eichler & Combretaceae & 2.0 & 0.3 & 20.0 & 0.9 & 0.0 & 0.1 & 1.2 & 0.4 \\
\hline Tocoyena formosa (Cham. \& Schltdl.) K. Schum. & Rubiaceae & 2.0 & 0.3 & 20.0 & 0.9 & 0.0 & 0.1 & 1.2 & 0.4 \\
\hline Platypodium elegans Vogel & Fabaceae & 2.0 & 0.3 & 20.0 & 0.9 & 0.0 & 0.1 & 1.2 & 0.4 \\
\hline Apuleia leiocarpa (Vogel) J.F. Macbr. & Fabaceae & 2.0 & 0.3 & 10.0 & 0.5 & 0.0 & 0.2 & 0.9 & 0.3 \\
\hline Eugenia bimarginata DC. & Myrtaceae & 2.0 & 0.3 & 10.0 & 0.5 & 0.0 & 0.2 & 0.9 & 0.3 \\
\hline Simira cf. corumbensis (Standl.) Steyerm. & Rubiaceae & 2.0 & 0.3 & 10.0 & 0.5 & 0.0 & 0.1 & 0.8 & 0.3 \\
\hline Matayba guianensis Aubl. & Sapindaceae & 1.0 & 0.1 & 10.0 & 0.5 & 0.0 & 0.2 & 0.8 & 0.3 \\
\hline Piper arboreum Aubl. & Piperaceae & 2.0 & 0.3 & 10.0 & 0.5 & 0.0 & 0.0 & 0.7 & 0.3 \\
\hline Trichilia elegans A. Juss. & Meliaceae & 1.0 & 0.1 & 10.0 & 0.5 & 0.0 & 0.2 & 0.7 & 0.2 \\
\hline Ximenia americana $\mathrm{L}$. & Olacaceae & 1.0 & 0.1 & 10.0 & 0.5 & 0.0 & 0.1 & 0.7 & 0.2 \\
\hline Guapira sp.2 & Nyctaginaceae & 1.0 & 0.1 & 10.0 & 0.5 & 0.0 & 0.1 & 0.7 & 0.2 \\
\hline Qualea multiflora Mart. & Vochysiaceae & 1.0 & 0.1 & 10.0 & 0.5 & 0.0 & 0.1 & 0.7 & 0.2 \\
\hline Copaifera langsdorffii Desf. & Fabaceae & 1.0 & 0.1 & 10.0 & 0.5 & 0.0 & 0.1 & 0.7 & 0.2 \\
\hline Diospyros hispida A.DC. & Ebenaceae & 1.0 & 0.1 & 10.0 & 0.5 & 0.0 & 0.1 & 0.6 & 0.2 \\
\hline Siphoneugena densiflora O.Berg & Myrtaceae & 1.0 & 0.1 & 10.0 & 0.5 & 0.0 & 0.0 & 0.6 & 0.2 \\
\hline Celtis iguanaea (Jacq.) Sarg. & Cannabaceae & 1.0 & 0.1 & 10.0 & 0.5 & 0.0 & 0.0 & 0.6 & 0.2 \\
\hline Endlicheria sp. & Lauraceae & 1.0 & 0.1 & 10.0 & 0.5 & 0.0 & 0.1 & 0.6 & 0.2 \\
\hline Genipa americana L. & Rubiaceae & 1.0 & 0.1 & 10.0 & 0.5 & 0.0 & 0.0 & 0.6 & 0.2 \\
\hline Cybistax antisyphilitica (Mart.) Mart. & Bignoniaceae & 1.0 & 0.1 & 10.0 & 0.5 & 0.0 & 0.0 & 0.6 & 0.2 \\
\hline Byrsonima pachyphylla A.Juss. & Malpighiaceae & 1.0 & 0.1 & 10.0 & 0.5 & 0.0 & 0.0 & 0.6 & 0.2 \\
\hline Rudgea viburnoides (Cham.) Benth. & Rubiaceae & 1.0 & 0.1 & 10.0 & 0.5 & 0.0 & 0.0 & 0.6 & 0.2 \\
\hline Myrcia tomentosa (Aubl.) DC. & Myrtaceae & 1.0 & 0.1 & 10.0 & 0.5 & 0.0 & 0.0 & 0.6 & 0.2 \\
\hline Sebastiania brasiliensis Spreng. & Euphorbiaceae & 1.0 & 0.1 & 10.0 & 0.5 & 0.0 & 0.0 & 0.6 & 0.2 \\
\hline Qualea grandiflora Mart. & Vochysiaceae & 1.0 & 0.1 & 10.0 & 0.5 & 0.0 & 0.0 & 0.6 & 0.2 \\
\hline Pouteria ramiflora (Mart.) Radlk. & Sapotaceae & 1.0 & 0.1 & 10.0 & 0.5 & 0.0 & 0.0 & 0.6 & 0.2 \\
\hline Cordia trichotoma (Vell.) Arráb. ex Steud. & Boraginaceae & 1.0 & 0.1 & 10.0 & 0.5 & 0.0 & 0.0 & 0.6 & 0.2 \\
\hline Schefflera cf. calva (Cham.) Frodin \& Fiaschi & Araliaceae & 1.0 & 0.1 & 10.0 & 0.5 & 0.0 & 0.0 & 0.6 & 0.2 \\
\hline Citrus sp. & Rutaceae & 1.0 & 0.1 & 10.0 & 0.5 & 0.0 & 0.0 & 0.6 & 0.2 \\
\hline Total & & 749.0 & 100.0 & 2220.0 & 100.0 & 16.5 & 100.0 & 300.0 & 100.0 \\
\hline
\end{tabular}


Table 4 - Woody community in one hectare of semi-deciduous seasonal forest in the municipality of Minaçu (GO). Decreasing Values of Importance (VI): D= Density (ind./ha), Do = Dominance ( $\left.\mathrm{m}^{2} / \mathrm{ha}\right), \mathrm{F}=$ Frequency $(\%), \mathrm{A}=$ absolute, $\mathrm{R}=$ relative.

\begin{tabular}{|c|c|c|c|c|c|c|c|c|c|}
\hline Scientific name & Family & DA & DR & FA & FR & DoA & DoR & VI & $\begin{array}{l}\text { VI } \\
(\%)\end{array}$ \\
\hline $\begin{array}{l}\text { Pseudobombax tomentosum (Mart. \& Zucc.) } \\
\text { A.Robyns }\end{array}$ & Malvaceae & 28.6 & 4.2 & 85.7 & 3.9 & 2.0 & 17.0 & 25.0 & 8.3 \\
\hline Dilodendron bipinnatum Radlk. & Sapindaceae & 65.7 & 9.5 & 100.0 & 4.5 & 1.0 & 8.6 & 22.6 & 7.6 \\
\hline Tabebuia roseoalba (Ridl.) Sandwith & Bignoniaceae & 77.1 & 11.2 & 100.0 & 4.5 & 0.7 & 6.2 & 21.9 & 7.3 \\
\hline Anadenanthera colubrina (Vell.) Brenan & Fabaceae & 34.3 & 5.0 & 100.0 & 4.5 & 0.9 & 7.2 & 16.7 & 5.6 \\
\hline Luehea paniculata Mart. \& Zucc. & Malvaceae & 21.4 & 3.1 & 85.7 & 3.9 & 0.9 & 7.6 & 14.6 & 4.9 \\
\hline Magonia pubescens A. St.-Hil. & Sapindaceae & 27.1 & 3.9 & 71.4 & 3.2 & 0.8 & 6.9 & 14.0 & 4.7 \\
\hline Callisthene fasciculata Mart. & Vochysiaceae & 25.7 & 3.7 & 71.4 & 3.2 & 0.8 & 6.3 & 13.3 & 4.4 \\
\hline Guettarda viburnoides Cham. \& Schltdl. & Rubiaceae & 42.9 & 6.2 & 85.7 & 3.9 & 0.4 & 3.2 & 13.2 & 4.4 \\
\hline Myracrodruon urundeuva Allemão & Anacardiaceae & 25.7 & 3.7 & 100.0 & 4.5 & 0.5 & 4.2 & 12.4 & 4.1 \\
\hline Aspidosperma subincanum Mart. & Apocynaceae & 31.4 & 4.6 & 85.7 & 3.9 & 0.4 & 2.9 & 11.4 & 3.8 \\
\hline Erythroxylum daphnites Mart. & Erythroxylaceae & 41.4 & 6.0 & 71.4 & 3.2 & 0.2 & 1.9 & 11.1 & 3.7 \\
\hline Campomanesia velutina (Cambess.) O.Berg & Myrtaceae & 38.6 & 5.6 & 85.7 & 3.9 & 0.2 & 1.6 & 11.0 & 3.7 \\
\hline Acrocomia aculeata (Jacq.) Lodd. ex Mart. & Arecaceae & 25.7 & 3.7 & 85.7 & 3.9 & 0.4 & 3.1 & 10.7 & 3.6 \\
\hline Eugenia dysenterica DC. & Myrtaceae & 28.6 & 4.2 & 71.4 & 3.2 & 0.3 & 2.4 & 9.8 & 3.3 \\
\hline Astronium fraxinifolium Schott ex Spreng. & Anacardiaceae & 18.6 & 2.7 & 100.0 & 4.5 & 0.3 & 2.3 & 9.5 & 3.2 \\
\hline Byrsonima pachyphylla A.Juss. & Malpighiaceae & 22.9 & 3.3 & 57.1 & 2.6 & 0.2 & 1.5 & 7.4 & 2.5 \\
\hline Cordia trichotoma (Vell.) Arráb. ex Steud. & Boraginaceae & 15.7 & 2.3 & 57.1 & 2.6 & 0.2 & 1.4 & 6.2 & 2.1 \\
\hline Dipteryx alata Vogel & Fabaceae & 7.1 & 1.0 & 57.1 & 2.6 & 0.3 & 2.6 & 6.2 & 2.1 \\
\hline Andira sp. & Fabaceae & 8.6 & 1.2 & 57.1 & 2.6 & 0.1 & 1.1 & 5.0 & 1.65 \\
\hline Terminalia argentea Mart. & Combretaceae & 8.6 & 1.2 & 42.9 & 1.9 & 0.2 & 1.7 & 4.9 & 1.6 \\
\hline Syagrus flexuosa (Mart.) Becc. & Arecaceae & 10.0 & 1.5 & 57.1 & 2.6 & 0.1 & 0.5 & 4.5 & 1.5 \\
\hline Swartzia acutifolia Vogel & Fabaceae & 5.7 & 0.8 & 57.1 & 2.6 & 0.1 & 0.7 & 4.1 & 1.4 \\
\hline $\begin{array}{l}\text { Pseudobombax longiflorum (Mart. \& Zucc.) A. } \\
\text { Robyns }\end{array}$ & Malvaceae & 4.3 & 0.6 & 28.6 & 1.3 & 0.1 & 0.9 & 2.8 & 0.9 \\
\hline $\begin{array}{l}\text { Tabebuia aurea (Silva Manso) Benth. \& Hook. f. } \\
\text { ex S. Moore }\end{array}$ & Bignoniaceae & 2.9 & 0.4 & 28.6 & 1.3 & 0.1 & 1.1 & 2.8 & 0.9 \\
\hline Jacaranda brasiliana (Lam.) Pers. & Bignoniaceae & 8.6 & 1.2 & 14.3 & 0.6 & 0.1 & 0.8 & 2.7 & 0.9 \\
\hline Agonandra brasiliensis Miers ex Benth. \& Hook.f. & Opiliaceae & 4.3 & 0.6 & 28.6 & 1.3 & 0.1 & 0.7 & 2.6 & 0.9 \\
\hline Copaifera langsdorffii Desf. & Fabaceae & 1.4 & 0.2 & 14.3 & 0.6 & 0.2 & 1.7 & 2.5 & 0.9 \\
\hline Myrcia sp. & Myrtaceae & 5.7 & 0.8 & 28.6 & 1.3 & 0.0 & 0.2 & 2.3 & 0.8 \\
\hline Pouteria macrophylla (Lam.) Eyma & Sapotaceae & 5.7 & 0.8 & 28.6 & 1.3 & 0.0 & 0.2 & 2.3 & 0.8 \\
\hline Cordia sp. & Boraginaceae & 5.7 & 0.8 & 28.6 & 1.3 & 0.0 & 0.2 & 2.3 & 0.76 \\
\hline Symplocos sp. & Symplocaceae & 4.3 & 0.6 & 28.6 & 1.3 & 0.0 & 0.3 & 2.2 & 0.7 \\
\hline Handroanthus impetiginosus (Mart. ex DC.) Mattos & Bignoniaceae & 1.4 & 0.2 & 14.3 & 0.6 & 0.2 & 1.3 & 2.2 & 0.7 \\
\hline Rhamnidium elaeocarpum Reissek & Rhamnaceae & 4.3 & 0.6 & 28.6 & 1.3 & 0.0 & 0.2 & 2.1 & 0.7 \\
\hline Byrsonima sp. & Malpighiaceae & 2.9 & 0.4 & 28.6 & 1.3 & 0.0 & 0.2 & 1.9 & 0.6 \\
\hline Psidium myrtoides O.Berg & Myrtaceae & 2.9 & 0.4 & 28.6 & 1.3 & 0.0 & 0.1 & 1.8 & 0.6 \\
\hline Cordiera sessilis (Vell.) Kuntze & Rubiaceae & 2.9 & 0.4 & 28.6 & 1.3 & 0.0 & 0.1 & 1.8 & 0.6 \\
\hline
\end{tabular}




\begin{tabular}{|c|c|c|c|c|c|c|c|c|c|}
\hline Scientific name & Family & DA & DR & FA & FR & DoA & DoR & VI & $\begin{array}{c}\text { VI } \\
(\%)\end{array}$ \\
\hline Simira cf. corumbensis (Standl.) Steyerm. & Rubiaceae & 2.9 & 0.4 & 14.3 & 0.6 & 0.0 & 0.1 & 1.1 & 0.4 \\
\hline Alibertia edulis (Rich.) A. Rich. & Rubiaceae & 1.4 & 0.2 & 14.3 & 0.6 & 0.0 & 0.3 & 1.1 & 0.4 \\
\hline Diospyros hispida A.DC. & Ebenaceae & 1.4 & 0.2 & 14.3 & 0.6 & 0.0 & 0.1 & 1.0 & 0.3 \\
\hline Roupala montana Aubl. & Proteaceae & 1.4 & 0.2 & 14.3 & 0.6 & 0.0 & 0.1 & 1.0 & 0.3 \\
\hline Eriotheca gracilipes (K. Schum.) A. Robyns & Malvaceae & 1.4 & 0.2 & 14.3 & 0.6 & 0.0 & 0.1 & 0.9 & 0.3 \\
\hline Kielmeyera coriacea Mart. \& Zucc. & Clusiaceae & 1.4 & 0.2 & 14.3 & 0.6 & 0.0 & 0.1 & 0.9 & 0.3 \\
\hline Leptolobium elegans Vogel & Fabaceae & 1.4 & 0.2 & 14.3 & 0.6 & 0.0 & 0.1 & 0.9 & 0.31 \\
\hline Tocoyena formosa (Cham. \& Schltdl.) K. Schum. & Rubiaceae & 1.4 & 0.2 & 14.3 & 0.6 & 0.0 & 0.0 & 0.9 & 0.3 \\
\hline Qualea grandiflora Mart. & Vochysiaceae & 1.4 & 0.2 & 14.3 & 0.6 & 0.0 & 0.0 & 0.9 & 0.3 \\
\hline Machaerium acutifolium Vogel & $\begin{array}{l}\text { Fabaceae } \\
\text { Faboideae }\end{array}$ & 1.4 & 0.2 & 14.3 & 0.6 & 0.0 & 0.1 & 0.9 & 0.3 \\
\hline Lafoensia pacari A. St.-Hil. & Lythraceae & 1.4 & 0.2 & 14.3 & 0.6 & 0.0 & 0.1 & 0.9 & 0.3 \\
\hline Aspidosperma pyrifolium Mart. & Apocynaceae & 1.4 & 0.2 & 14.3 & 0.6 & 0.0 & 0.1 & 0.9 & 0.3 \\
\hline Cheiloclinium cognatum (Miers) A.C. Sm. & Celastraceae & 1.4 & 0.2 & 14.3 & 0.6 & 0.0 & 0.0 & 0.9 & 0.3 \\
\hline Total & & 688.6 & 100.0 & 2228.6 & 100.0 & 12.0 & 100.0 & 300.0 & 100.0 \\
\hline
\end{tabular}

out in the fragments in the valley of Paranã (Silva \& Scariot 2003, 2004, 2004a; Scariot \& Sevilha 2005) suggest links with the seasonal forests of the Caatinga biome in the vegetation defined as "Caatinga Arbórea" (Santos et al. 2012), which has a high frequency of species such as Anadenanthera colubrina, Aspidosperma pyrifolium, Myracrodruon urundeuva and Handroanthus impetiginosus, besides other examples.

The species richness of the forest fragments was similar to a wide range of variation for this type of tropical ecosystem (Murphy \& Lugo 1986; Gillespie et al. 2000), which occupies different Brazilian biomes such as the Cerrado, Caatinga and the Atlantic Forest. As regards the sampling in Palmeirópolis, the Chao and Jackknife richness estimators reported values higher than the observed richness. Krebs (1989) and Hellmann \& Fowler (1999) suggested that, for samples smaller than 20 plots, the variations of the Jackknife method showed a better performance than estimates from the Bootstrap method, which provides more trustworthy data in larger samples. However, the results of the Bootstrap method were more similar to the observed richness of the current study.

The values the Shannon diversity index and evenness were lower than the diversity recorded in many preserved semi-deciduous forests in other regions in Brazil (Silva et al. 2003; Silva \& Araújo 2009) and also presented lower values than those in semi-deciduous forests with some degree of human disturbance in the past (Araújo \& Haridasan 1997; Garcia et al. 2011). However, the observed values of the Shannon diversity index and evenness are included in the wide range of variation found in surveys in intact and human-disturbed semi-deciduous and deciduous forests, being higher than the records from humandisturbed semi-deciduous forests (Fonseca \& Rodrigues 2000). The evenness in both fragments did not indicate a powerful dominance of a few species over others, suggesting a relatively stability in the species distribution.

The occurrence of cattle, the abundance of Guadua paniculata, the evidence of fire inside the fragments and the logging observed in the field seem to have provided conditions for lianas to become abundant, as one could see in other South American seasonal forests (Fredericksen \& Mostacedo 2000). The liana tangles can reduce the natural regeneration process in forest formations for years (Schnitzer et al. 2000; Tabanez \& Viana 2000). In the deciduous forests of the Paranã valley, Vieira \& Scariot (2006b) reported a liana occupation of $22 \%$ in a disturbed area, whereas preserved areas showed $6 \%$ of occupation. Both studied fragments showed especially high values for liana tangle frequency, which is typical of disturbed environments (Oliveira-Filho et al. 1997; Vieira \& Scariot 2006b). 
The values of density, volume and dominance/ basal area were included in the typical range of seasonal forests and were even higher than in some less disturbed deciduous forests (Scariot \& Sevilha 2005). The comparison with these forests indicates the variability of density and basal area and that the studied fragments were a little denser and with more dominance than the deciduous forests in Table 2. However, the values were lower than other semi-deciduous forests from Goiás, Minas Gerais and São Paulo. Haidar et al. (2005) reported density values of 1097.8 ind.ha $^{-1}$ in centralsouthern Goiás. Also in southern Goiás, Garcia et al. (2011) reported slightly lower density values, at between 882 and 1039 ind.ha ${ }^{-1}$. These authors also calculated the value of $11.1 \mathrm{~m}^{2}$.ha ${ }^{-1}$ for the basal area in semi-deciduous forest fragments in early regeneration stage, which is near the value recorded in Minaçú. Araújo \& Haridasan (1997) indicated density values higher than 1800 ind.ha $^{-1}$ and 2500 ind.ha ${ }^{-1}$ for two forest fragments in Uberlândia. In São Paulo, Ivanauskas et al. (2002) reported density of 2125 ind ha $^{-1}$ and a high value of $45.22 \mathrm{~m}^{2}$.ha $\mathrm{h}^{-1}$ for basal area. The results of the current study suggest the fragments of seasonal forests in northeastern Goiás were structurally closer to the deciduous forests in Goiás recorded in the Paranã river valley than to the semi-deciduous forests sampled in areas located in the southern.

In spite of the high number of dead standing individuals in both studied forest fragments, these values were similar to those recorded in other surveys in a number of regions (Ivanauskas \& Rodrigues 2000; Ivanauskas et al. 2002; Silva \& Soares 2002; Lopes et al. 2002; Silva \& Scariot 2003; Haidar et al. 2005). So this variable did not add information about possible patterns of the community structure.

Several species, such as Anadenanthera colubrina, Aspidosperma pyrifolium, Aspidosperma subincanum, Dilodendron bipinnatum, Hymenaea courbaril, Myracrodruon urundeuva, Pseudobombax tomentosum, Handroanthus impetiginosus and Tabebuia roseoalba also showed high values of importance in deciduous forest located in the Paranã river valley (Silva \& Scariot 2003; 2004; 2004a; Nascimento et al. 2004; Scariot \& Sevilha 2005). Are these results related to the geographic distance? Is it a remnant of the fragmentation in the former "mato grosso goiano"? With the current available data it is not possible to reach a final conclusion. Furthermore, the species
A. colubrina, D. bipinnatum, $H$. courbaril and $M$. urundeuva also stood out in the semi-deciduous forest from central Goiás (Haidar et al. 2005) in spite of the lower similarity and higher geographic distance. These species were also highlighted by Oliveira-Filho et al. (2006), showing a high frequency on checklists from low-altitude tropical seasonal forests in the central-western region of Brazil. In particular, the value for A. colubrina, the first IV in Palmeirópolis and the fourth in Minaçú, is interesting given the high frequency of this species in Central Brazil, and which was not considered in past studies (Prado \& Gibbs 1993; Pennington et al. 2000; Prado 2000) because of the lack of published data.

The small and human-disturbed forest fragments studied showed composition and structure that are typical of low-altitude seasonal forests in the middle/upper Tocantins river basin. The floristic composition was more similar to that of a deciduous seasonal forest in the Paranã valley, and more dissimilar to other forests in this same valley. This result and a higher dissimilarity related to the southern forests in Goiás suggest limitations on dispersion of the woody flora and a particular flora in each fragment with relatively similar structure and diversity. These floristically heterogeneous forest fragments are the last remnants of that endangered forest physiognomy in this region of Goiás.

\section{Acknowledgments}

The authors would like to thank Glocimar Pereira da Silva, Isabela Lustz Portela Lima, João Benedito Pereira, Juarez Amaral and Gledson Alves Moreira for field assistance and Susan Casement for English review. This research was supported by Consórcio de Energia São Salvador (CESS) and Fundação Arthur Bernardes (FUNARBE)

\section{References}

APG III. 2009. An update of the Angiosperm Phylogeny Group classification for the orders and families of flowering plants: APG III. Botanical Journal of the Linnean Society 161: 105-121.

Araújo, G.M. \& Haridasan, M. 1997.Estrutura fitossociológica de duas matas mesófilas semidecíduas, em Uberlândia, triângulo mineiro. Naturalia 22: 115-129.

BRASIL. 1981. Projeto Radambrasil. Folha SD-22 Goiás; geologia, geomorfologia, pedologia, vegetação e uso potencial da terra. Ministério de Minas e Energia, Rio de Janeiro. 28p. 
Botrel, R.T.; Oliveira-Filho, A.T.; Rodrigues, L.A. \& Curi, N.. 2002. Influência do solo e topografia sobre as variações da composição florística e estrutura da comunidade arbóreo-arbustiva de uma floresta estacional semidecidual em Ingaí, MG. Revista Brasileira de Botânica 25: 195-213.

CDB. 2010. Panorama da Biodiversidade Global 3. Secretariado da Convenção sobre Diversidade Biológica. Ministério do Meio Ambiente, Brasília. 94p.

Carvalho, W.A.C.; Oliveira-Filho, A.T.; Fontes, M.A.L. \& Curi, N. 2007. Variação espacial da estrutura da comunidade arbórea de um fragmento de floresta semidecídua em Piedade do Rio Grande, MG, Brasil. Revista Brasileira de Botânica 30: 315-335.

Cestaro, L.A. \& Soares, J.J. 2004. Variações florística e estrutural e relações fitogeográficas de um fragmento de floresta decídua no Rio Grande do Norte, Brasil. Acta Botanica Brasilica 18: 203-218.

CIENTEC. 2004. Mata nativa. Sistema para análise fitossociológica e elaboração de planos de manejo de florestas nativas. Viçosa.

Espírito-Santo, M.M.; Sevilha, A.C.; Anaya, F.C.; Barbosa, R.; Fernandes, G.W.; Sanchez-Azofeifa, G.A.; Scariot, A.; Noronha, S.E. \& Sampaio, C.A. 2009. Sustainability of tropical dry forests: two case studies in southeastern and central Brazil. Forest Ecology and Management 258: 922-930.

Faissol, S. 1953. Alguns aspectos do mato grosso de Goiás. Boletim Goiano de Geografia 11: 63-83.

Fonseca, R.C.B. \& Rodrigues, R.R. 2000. Análise estrutural e aspectos do mosaico sucessional de uma floresta semidecidua em Botucatu, SP. Scientia Forestalis 57: 27-43.

Fredericksen, T.S. \& Mostacedo, B. 2000. Regeneration of timber species following selection logging in a Bolivian tropical dry forest. Forest Ecology and Management 131: 47-55.

Garcia, P.O.; Valente, A.S.M.; Pifano, D.S.; Pessoa, J.F.S.; Busato, L.C.; Fontes, M.A.L. \& OliveiraFilho, A.T. 2011. Species composition and floristic relationship in southern Goiás forest enclaves. Rodriguésia 61: 123-137.

Gillespie, T.W.; Grijalva, A. \& Farris, C.N. 2000.Diversity, composition and structure of tropical dry forests in Central America. Plant Ecology 147: 37-47.

Gotelli, N.J. \& Colwell, R.K. 2001. Quantifying biodiversity: procedures and pitfalls in the measurement and comparison of species richness. Ecology Letters 4: 379-391.

Haidar, R.F.; Felfili, J.M.; Pinto, J.R.R. \& Fagg, C.W. 2005. Fitossociologia da vegetação arbórea em fragmentos de floresta estacional, no Parque Ecológico Altamiro de Moura Pacheco, GO. Boletim do HerbárioEzechias Paulo Heringer 15: 19-46.
Hellmann, J.J. \& Fowler, G.W. 1999. Bias, precision and accuracy of four measures of species richness. Ecological Applications 9: 824-834.

IBGE. 2012. Manual técnico da vegetação brasileira. $2^{\mathrm{a}}$ ed. Instituto Brasileiro de Geografia e Estatística, Rio de Janeiro. 275p.

Imaña-Encinas, J.; Macedo, L.A. \& Paula, J.E. 2007. Florística e fitossociologia de um trecho de floresta estacional semidecidual na área do ecomuseu do Cerrado em Pirenópolis-Goiás. Cerne 13: 308-320.

Ivanauskas, N.M. \& Rodrigues, R.R. 2000. Florística e fitossociologia de remanescentes de floresta estacional decidual em Piracicaba, São Paulo, Brasil. Revista Brasileira de Botânica 23: 291-304.

Ivanauskas, N.M.; Rodrigues, R.R. \& Nave, A.G. 2002. Fitossociologia de um remanescente de floresta estacional semidecidual em Itatinga-SP, para fins de restauração de áreas degradadas. Revista Árvore 26: 43-57.

Janzen, D.H. 1997. Florestas tropicais secas: o mais ameaçado dos grandes ecossistemas tropicais. In: Wilson, E.O. \& Peter, F.M. (orgs.). Biodiversidade. Nova Fronteira, Rio de Janeiro. Pp. 166-176.

Krebs, C.J. 1989. Ecological methodology. Addison Wesley Longman, Petaluma. 624p.

Kunz, S.H.; Martins, V.A.; Ivanauskas, M.N.; Silva, E. \& Stefanello, D. 2010.Estrutura fitossociológica de um trecho de Floresta Estacional Perenifólia, bacia do rio das Pacas, Querência - MT. Cerne 16: 115-122.

Legendre, P. \& Legendre, L. 2012. Numerical ecology. $2^{\text {nd }}$ ed. Elsevier Science, Amsterdam. 1006p.

Lima, M.S.; Damasceno-Junior, G.A \& Tanaka, M.O. 2010. Aspectos estruturais da comunidade arbórea em remanescentes de floresta estacional decidual em Corumbá, MS, Brasil. Revista Brasileira de Botânica 33: 437-453.

Lopes, W.P.; Silva, A.F.; Souza, A.L. \& Meira Neto, J.A.A. 2002. Estrutura fitossociológica de um trecho de vegetação arbórea no Parque Estadual do Rio Doce - Minas Gerais, Brasil. Acta Botanica Brasilica 16: 443-456.

Magurran, A.E. \& McGill, B.J. 2011. Biological diversity: frontiers in measurement and assessment. Oxford University Press, Oxford. 345p.

Mueller-Dubois, D.Y. \& Ellemberg, M. 1974. Aims and methods in vegetation ecology. Willey and Sons, New York. 547p.

Murphy, P.G. \& Lugo, A.E. 1986. Ecology of tropical dry forest. Annual Review of Ecology and Systematics17: 67-88.

Nascimento, L.M. \& Rodal, M.J.N. 2008. Fisionomia e estrutura de uma floresta estacional montana do maciço da Borborema, Pernambuco - Brasil. Revista Brasileira de Botânica 31: 27-39. 
Nascimento, A.T.; Felfili, J.M. \& Meirelles, E.M. 2004. Florística e estrutura da comunidade arbórea de um remanescente de floresta estacional decidual de encosta, Monte Alegre, GO, Brasil. Acta Botanica Brasilica 18: 659-669.

Oksanen, J. 2011. Vegan: community ecology package. $\mathrm{R}$ package version 1.17-11. Available in <http:// CRAN.R-project.org/package $=$ vegan $>$. Access on 10 Oct 2009.

Oliveira-Filho, A.T.; Mello, J.M. \& Scolforo, J.R. 1997. Effects of past disturbance and edges on tree community structure and dynamics within a fragment of tropical semideciduous forest in southeastern Brazil over a five-year period (1987-1992). Plant Ecology 131: 45-66.

Oliveira-Filho, A.T.; Jarenkow, J.A. \& Rodal, M.J. 2006. Floristic relationships of seasonally dry forests of eastern south America based on tree species distribution patterns. In: Pennington, R.T.; Lewis, G.P. \& Ratter, J.A. (orgs.). Neotropical savannas and seasonally dry forests: plant diversity, biogeography, and conservation. Taylor \& Francis, New York. Pp. 159-192.

Palmer, M.W. 1990. The estimation of species richness by extrapolation. Ecology 71: 1195-1198.

Pennington, R.T.; Prado, D.E. \& Pendry, C.A. 2000. Neotropical seasonally dry forests and quaternary vegetation changes. Journal of Biogeography 27 : 261-273.

Pennington, R.T.; Lewis, G.P. \& Ratter, J.A. 2006. An overview of the plant diversity, biogeography and conservation of neotropical savannas and seasonally dry forests. In: Pennington, R.T.; Lewis, G.P. \& Ratter, J.A. (orgs.). Neotropical savannas and seasonally dry forests: plant diversity, biogeography and conservation. Taylor \& Francis, New York. Pp.1-29.

Pereira, I.M.; Andrade, L.A.; Barbosa, M.R.V. \& Sampaio, E.V.S.B. 2002. Composição florística e análise fitossociológica do componente arbustivoarbóreo de um remanescente florestal no agreste Paraibano. Acta Botanica Brasilica 16: 357-369.

Prado, D.E. \& Gibbs, P.E. 1993. Patterns of species distribution in the dry seasonal forests of South America. Annals of the Missouri Botanical Garden 80: 902-927.

Prado, D.E. 2000.Seasonally dry forests of tropical south america: from forgotten ecosystem to a new phytogeographic unit. Edinburgh Journal of Botany 57: 437-461.

R Development Core Team. 2011. R: a language and environment for statistical computing. R Foundation for Statistical Computing, Viena.

Ribeiro, J.F. \& Walter, B.M.T. 2008. As principais fitofisionomias do bioma Cerrado. In: Sano, S.M.; Almeida, S.P. \& Ribeiro, J.F. (orgs.). Cerrado: ecologia e flora. Vol. 1. Embrapa Cerrados/Embrapa Informação Tecnológica, Brasília. Pp. 151-212
Salis, S.M.; Silva, M.P.; Mattos, P.P.; Silva, J.S.V.; Pott, V.J. \& Pott, A. 2004. Fitossociologia de remanescentes de floresta estacional decidual em Corumbá, estado do Mato Grosso do Sul, Brasil. Revista Brasileira de Botânica 27: 671-684.

Santos, M.R.; Oliveira-Filho, A.T.; Eisenlohr, P.V.; Queiroz, L.P.; Cardoso, D.B.O.S. \& Rodal. M.J.N. 2012. Identity and relationships of the arboreal Caatinga among other floristic units of seasonally dry tropical forests (SDTFs) of north-eastern and Central Brazil. Ecology and Evolution 2: 409-428.

Scariot, A. \& Sevilha, A.C. 2000. Diversidade, estrutura e manejo de florestas deciduais e as estratégias para a conservação. In: Cavalcanti, T.B. \& Walter, B.M.T. (orgs.). Tópicos atuais em botânica. $51^{\circ}$ Congresso Nacional de Botânica. Sociedade Botânica do Brasil. Embrapa Recursos Genéticos e Biotecnologia, Brasília. Pp. 183-188.

Scariot, A. \& Sevilha, A.C. 2005. Biodiversidade, estrutura e conservação de florestas estacionais deciduais no Cerrado. In: Scariot, A.; Souza-Filho, J.C. \& Felfili, J.M. (orgs.). Cerrado: ecologia, biodiversidade e conservação. Ministério do Meio Ambiente, Brasília. Pp. 121-140.

Schnitzer, S.; Dalling, J. \& Carson, W. 2000. The impact of lianas on tree regeneration in tropical forest canopy gaps: evidence for an alternative pathway of gap-phase regeneration. Journal of Ecology 88: 655-666.

SEPLAN. 2008. Atlas do Tocantins: subsídios ao planejamento da gestão territorial. Secretaria de Planejamento, Palmas. 62p.

SIEG. 2010. Sistema de Informações Estatística e Geográficas de Goiás. Available in <http://www. sieg.go.gov.br>. Access on 8 Nov 2010.

Silva, A.F.; Oliveira, R.V.; Santos, N.R.L. \& Paula, A. 2003. Composição florística e grupos ecológicos das espécies de um trecho de floresta semidecíduasubmontana da Fazenda São Geraldo, Viçosa, MG. Revista Árvore 27: 311-319.

Silva, L.A. \& Scariot, A.O. 2003. Composição florística, estrutura da comunidade arbórea em floresta estacional decidual em afloramento calcário (Fazenda São José, São Domingos, GO, bacia do rio Paranã). Acta Botanica Brasilica 17: 305-313.

Silva, L.A. \& Scariot, A.O. 2004. Comunidade arbórea de uma floresta estacional decídua sobre afloramento calcário na Bacia do rio Paranã. Revista Árvore 28: 61-67.

Silva, L.A. \& Scariot, A.O. 2004a. Composição e estrutura da comunidade arbórea de uma floresta estacional decidual sobre afloramento calcário no Brasil Central. Revista Árvore 28: 69-75.

Silva, L.A. \& Soares, J.J. 2002. Levantamento fitossociológico de um fragmento de floresta estacional semidecídua, no município de São Carlos, SP. Acta Botanica Brasilica 16: 205-216. 
Silva, F.A.M.; Assad, E.D. \& Evangelista, B.A. 2008. Caracterização climática do bioma Cerrado. In: Sano, S.M.; Almeida, S.P. \& Ribeiro, J.F. (orgs.) Cerrado: ecologia e flora. Vol. 1. Embrapa Cerrados/Embrapa Informação Tecnológica, Brasília. Pp. 70-88.

Silva, M.R. \& Araújo, G.M. 2009. Dinâmica da comunidade arbórea de uma floresta semidecidual em Uberlândia, MG, Brasil. Acta Botanica Brasilica 23: 49-56.

Silva-Júnior, M.C.; Barros, N.F. \& Cândido, J.F. 1987. Relações entre parâmetros de solo e da vegetação de cerrado na Estação Florestal de Experimentação de Paraopeba-MG. Revista Brasileira de Botânica 10: 125-137.

Siqueira, A.S.; Araújo, G.M. \& Schiavini, I. 2009. Estrutura do componente arbóreo e características edáficas de dois fragmentos de floresta estacional decidual no vale do rio Araguari, MG, Brasil. Acta Botanica Brasilica 23: 10-21.

Sobral, M.; Proença, C.; Souza, M.; Mazine, F. \& Lucas, E. 2012. Myrtaceae. In: Lista de espécies da flora do Brasil. Jardim Botânico do Rio de
Janeiro. Disponível em $<$ http://floradobrasil.jbrj. gov.br/2012/FB010759>. Acesso em 20 Mar 2012.

Tabanez, A.A.J. \& Viana, V.M. 2000. Path structure within Brazilian Atlantic forest fragments and implications for conservation. Biotropica 32: 925-933.

Veloso, H.P. 1963. Os grandes clímaces do Brasil III: considerações gerais sobre a vegetação da região centro-oeste. Memórias do Instituto Oswaldo Cruz 61: 357-370.

Vieira, D.L.M. \& Scariot, A. 2006a. Principles of natural regeneration of tropical dry forest for restoration. Restoration Ecology 14: 11-20.

Vieira, D.L.M. \& Scariot, A. 2006b. Effects of logging, liana tangles and pasture on seed fate of dry forest tree species in Central Brazil. Forest Ecology and Management 230: 197-205.

Whitmore, T.C. 1997. Tropical forest disturbance, disappearance and species loss. In: Laurance, W.F. \& Bierregaard, R.O. (orgs.). Tropical forest remnants: ecology, management, and conservation of fragmented communities. The University of Chicago Press, Chicago. Pp. 3-12. 